\title{
Effect of Mineral Diatomaceous Earth-based Drugs on Adaptive Reactions in Weaned Pigs
}

\section{Aleksey Savinkov1, Pavel Iliasov², Olga Guseva², Kurlykova Julya1, and Aleksandr Mescheriakov ${ }^{1}$}

${ }^{1}$ Samara state agrarian University Federal State Budgetary Educational Institution of Higher Education, Samara, Russia

${ }^{2}$ Samara Research Veterinary Institute - Branch of Federal Research Center for Virology and Microbiology, Samara, Russia

\section{Abstract}

The effects of diatomaceous earth-based drugs, Diatomite and Biokoretron, on morphofunctional parameters, blood chemistry, antibody mediated non-specific immunity and growth of post-weaning pigs were investigated. The studies showed that both drugs had growth promoting and antianemic effects on the weaned pigs, increased the protein and mineral metabolism rate, and stimulated non-specific immunity factors.

Corresponding Author:

Aleksey Savinkov

a_v_sav@mail.ru

Received: 25 October 2019

Accepted: 15 November 2019

Published: 25 November 2019

Publishing services provided by Knowledge E

(c) Aleksey Savinkov et al. This article is distributed under the terms of the Creative Commons

Attribution License, which

permits unrestricted use and redistribution provided that the original author and source are credited.

Selection and Peer-review under the responsibility of the AgroSMART 2019 Conference Committee.

\section{Introduction}

The improvements in beef cattle breeding and pork production in the Russian Federation acquires the status of the strategic development of the national food base in light of import substitution program. Pig breeding plays an important role in providing the population with meat [1]. However, due to the atmosphere and planet surface pollution with industrial wastes, feed and feed supplements used in animal husbandry often contain toxic substances (heavy metals, pesticide residues, etc.), which are potentially harmful for the animals. Such feeding leads to metabolic disorders, various diseases and depresses animal reproductive functions [2].

Among farm animals, pigs are most susceptible to the lack of certain nutrients, with special attention given to mineral substances. Mineral deficiency leads to metabolic disorders, functional deterioration of the major body systems and emergence of various diseases [3].

In recent years, Russian and foreign researchers have demonstrated that enterosorS OPEN ACCESS 
was found that the enterosorbent supplementation of the diets of young pigs with internal pathologies promotes healing through normalization of metabolic processes and improvements in natural resistance [8]. Natural mineral sorbent-based drugs contribute to slower passage of the digestive tract contents by sorbing exo- and endotoxins with following evacuation from the body [9--11]. The intestine fermentation and putrefaction rate is reduced, therefore improving the digestive process. The marked ion-exchange properties of these drugs promote an extensive intake of macronutrients and trace elements [5].

In a wide variety of mineral based drugs, diatomaceous earth has a special position because its formation occurred as a result of mineralization of the organic matrix of ancient vegetation. Diatomaceous earth is a natural mineral that appears as a loose, light finely porous rock mostly composed of tiny $(0.0--0.04 \mathrm{~mm})$ opal skeletons of diatoms. Its average density is typically less than $100 \mathrm{~kg} / \mathrm{m} 3$, the porosity is $70--75 \%$. Diatomaceous earth is white, yellow, and sometimes dark gray and brownish-gray. It contains soluble silica (80--87 \%), iron oxide (3.2 \%), aluminum oxide (5--7\%), calcium oxide (0.7--1.2 \%), zinc, copper, and manganese chelates $(0.043 \%)$, betaine $(0.48 \%)$ and impurities such as clay, sand, and glauconite.

Diatomite (diatomaceous earth fine powder) and Biokoretron drugs were manufactured using diatomaceous earth from Inza deposit, Ulyanovsk region, Russia, at Diatom Integrated Plant LLC.

Biokoretron comprises heat-treated diatomaceous earth (97\%). It also contains BacilIus licheniformis and Bacillus subtilis-based additives, and ester oil complex. It belongs to natural adsorbents, is environmentally friendly and meets the requirements for this type of products.

Given the multifactorial positive biological effects associated with diatomaceous earth based drugs, their safety and low cost, as well as insufficient information regarding them, we set the following objective of the study.

The study was aimed to improve the measures intended to elevate the adaptation stability of young pigs in the large-scale enterprise environment.

The study scope included investigation of the effect of Diatomite and Biokoretron drugs on morpho-functional parameters, blood chemistry and antibody mediated nonspecific immunity and growth of post-weaning pigs. 


\section{Materials and Methods}

\subsection{Research subject}

An industrial experiment within the Diatomite and Biokoretron trial was performed at Komsomolets LLC pig farm, Kinel district, Samara region, Russia.

Effect of drug on morphological and functional characteristics was studied in 1--1.5month healthy pigs within 30 days. Experiment design involved use of 30 animals randomized to 3 groups of 10 animals: Group 1 received Diatomite (diatomaceous earth fine powder) in pure form; Group 2 received another diatomaceous earth based drug, Biokoretron; and Group 3 represented the control and received a diet according the feeding regimen established at the farm. The diet was mainly based on fodder composed of wheat ( $40 \%$ ) and barley (60\%). Experimental drugs were administered daily during the morning and evening feeding assuming $1 \mathrm{~g} / \mathrm{kg}$ daily feed.

The blood samples were taken on the Days 1, 10 and 30 for subsequent CBC, blood chemistry and immunological studies. Subject animals' health, survival and growth rate were monitored throughout the experiment. Body weight was assessed at the baseline and at the end of the study.

Hematology and blood chemistry tests were performed using Celly 70 veterinary hematology system (BioCode--Hycel, France SA) and Mindray BS-380 chemistry analyzer (Mindray, China) with commercial kits.

To evaluate serum non-specific resistance, we determined bactericidal activity using Escherichia coli O111 test culture, and lysozyme activity using 1-day culture of Micrococcus luteus 2665 strain obtained from Scientific Centre for Expert Evaluation of Medicinal Products. Statistical processing of the data obtained was performed using Microsoft Office Excel 2010.

\section{Results}

The analysis of obtained results suggested a positive effect of the drugs tasted on the animal body weight gain. Thus, pig weight at baseline was of $15.0 \pm 0.9 \mathrm{~kg}$ in the Group $1,15.3 \pm 1.1 \mathrm{~kg}$ in the Group 2 and $15.1 \pm 1.0 \mathrm{~kg}$ in controls. As early as on Day 10, body weight gain was $29.3 \%(p<0.01)$ and $38.2 \%(p<0.01)$ in the Groups 1 and 2 vs. control group, respectively. At the end of the experiment, these differences were of $40.4 \%$ ( $p$ $<0.001)$ and $45.7 \%(p<0.001)$, respectively. 
Therefore, in the Group 1 where the animals received feed supplemented with Diatomite, body weight increased by $11.6 \mathrm{~kg}$ within 30 days; in the group receiving Biokoretron, the gain was of $11.1 \mathrm{~kg}$. In the control group, the weight gain was only 3.7 $\mathrm{kg}$, i.e. three times less than in the treatment groups.

Thus, Diatomite and Biokoretron have a marked effect on the animal growth rate.

Analysis of the laboratory findings indicated that both pure diatomaceous earth and Biokoretron have a positive effect on the animal homeostasis parameters.

When evaluating blood hematology regarding $\mathrm{RBC}$, it was revealed that animals in Group 1, Group 2 and control group had reduced RBC count $(5.9 \pm 0.2,5.4 \pm 0.3$, and $\left.5.7 \pm 0.6 \times 10^{12} / \mathrm{L}\right), \mathrm{Hb}(75.4 \pm 2.7,75.0 \pm 3.3$, and $74.0 \pm 5.4 \mathrm{~g} / \mathrm{L})$, and hematocrit values $(27.1 \pm 0.7 \%, 27.8 \pm 1.3 \%$, and $26.7 \pm 2.3 \%)$ at the baseline. By the end of experiment, Group 1 receiving Diatomite demonstrated $9.5 \%$ increase in the RBC count vs. the control group $(\mathrm{p}<0.05)$. Together with an increase in hemoglobin by $17.8 \%$ and hematocrit by $19.9 \%$, this suggests the beneficial effect of Diatomite on hematopoiesis, which ultimately enhances redox reactions in the tissues and improves metabolism. In addition, the parameters used to assess the clinical anemias, mean corpuscular volume, mean corpuscular hemoglobin and mean corpuscular hemoglobin concentration were $5.9 \%, 8.2 \%$ and $3.0 \%$ higher in Group 1 vs. control, respectively, which also confirms the stimulating effect of Diatomite on erythropoiesis and hematopoiesis.

Group 2 (pigs receiving Biokoretron) was not able to demonstrate any significant difference in RBC parameters vs. controls. Thus, the drug has no stimulating effect on erythropoiesis in this period, and all the changes revealed were within the physiological range.

Baseline WBC count in all groups was at the upper normal level $\left(22.0--22.1 \times 10^{9} / \mathrm{L}\right)$, but this is a known effect observed in young animals in the presence of a common set of stress factors during weaning. After treatment, a decrease in this parameter by 26.8 and $20.0 \%$ from the baseline was found in Groups 1 and 2, respectively; the resulting values were within the physiological range. However, in these groups WBC was 10.2 $\%(p<0.05)$ and $3.0 \%$ higher vs. the control group, respectively, which can indirectly indicate a higher level of non-specific immune cell activity. WBC differential suggested no abnormalities or fluctuations in WBC type counts and percentages during the study.

Protein metabolism assessment revealed that all the groups had a reduced total protein content at the baseline $(45.58 \pm 3.92,46.02 \pm 1.26$, and $45.52 \pm 4.79 \mathrm{~g} / \mathrm{L}$ in Group 1, Group 2 and control group, respectively), which in this case is typical of nutritional protein metabolism disorders. Subsequently, a dynamic increase in this parameter was observed in all groups. However, the animals in treatment groups had 
higher protein level than the intact counterparts. At the end of the experiment, total protein was significantly increased by $3.0 \%$ in Group 1 (Diatomite) and by $7.2 \%$ in Group 2 (Biokoretron) vs. controls $(p<0.05)$. These conformed to the changes in albumin protein fraction. By Day 30, albumin level in the Groups 1 and 2 exceeded the control value by 1.2 and $18.3 \%$, respectively $(p<0.05)$.

A similar trend was observed with respect to urea level. Urea production is considered a very stable liver function that characterizes both the liver status and the protein metabolism rate. Our studies allowed revealing that by the end of the experiment Group 2 demonstrated $3.7 \%$ higher urea level vs. the control group $(p<0.05)$ while in Group 1 this parameter remained at the control level.

Thus, the use of diatomaceous earth based drugs stimulated liver metabolic activity that resulted in an increase in protein production and utilization.

When assessing mineral metabolism in pigs, it can be noted that the calcium content was significantly lower than normal range in all groups. Its value was of $1.44 \pm 0.27$ $\mathrm{mmol} / \mathrm{L}$ in Group 1, $1.51 \pm 0.12 \mathrm{mmol} / \mathrm{L}$ in Group 2, and $1.46 \pm 0.08 \mathrm{mmol} / \mathrm{L}$ in control group. Baseline phosphorus levels were within the reference range $(2.04 \pm 0.29,2.15$ \pm 0.10 , and $1.98 \pm 0.11 \mathrm{mmol} / \mathrm{L}$ in Group 1 , Group 2 and control group, respectively). As a result, there was an abnormal calcium-phosphorus ratio of $0.7,0.5$ and 0.6 in Group 1, Group 2 and control group, respectively, that was due to low calcium and optimal phosphorus levels. Alkaline phosphatase activity was at the upper limit of the reference range $(195.8 \pm 33.7,198.2 \pm 23.7$, and $201.0 \pm 31.7 \mathrm{IU} / \mathrm{L}$ in Group 1, Group 2 and control group, respectively). Thus, the experimental animals had changes characteristic of rickets.

Throughout the experiment, total calcium level dynamically increased in all groups due to common practice established at the farm, however, the animals receiving the drug had more marked changes. At the end of the study, serum calcium concentrations were $1.99 \pm 0.14,2.08 \pm 0.15$, and $1.85 \pm 0.16 \mathrm{mmol} / \mathrm{L}$ in Group 1, Group 2 and control group, respectively. Thus, calcium level was $7.6 \%$ higher in the Diatomite treated group and $12.4 \%$ higher $(p<0.05)$ in Biokoretron treated group vs. controls.

Serum phosphorus dynamics was also positive in all treated animals. By the end of experiment, the phosphorus levels was $30.8 \%$ and $25.5 \%$ higher in Group 1 and Group 2 vs. the control values, respectively, but these differences were not statistically significant.

Calcium-phosphorus ratio significantly changed vs. baseline and was of 1:1 in all groups. 
Alkaline phosphatase activity increased in all groups throughout the experiment and reached maximum values by Day 30 (436.0 $\pm 46.1,459.0 \pm 16.5$, and $284.0 \pm 70.1 \mathrm{IU} / \mathrm{L}$ in Group 1, Group 2 and control group, respectively), exceeding the control value by 53.1 and 61.3 \% in Group 1 and Group 2. Alkaline phosphatase is produced by liver, bone tissue, intestine, placenta, and kidneys; in this case, the increase in alkaline phosphatase activity was driven by bones because serum calcium didn't reach the lower normal limit by the end of study. The alkaline phosphatase is known to stimulate osteoblast activity, and this supports the activation of osteoplastic process in piglets receiving the experimental drugs. Thus, all the changes indicated an increased mineral metabolism rate in treatment groups.

Iron is involved in redox reactions and is an essential part of hemoglobin. Optimal blood iron levels create the necessary conditions for improving the hematopoiesis.

Throughout the experiment, iron concentration was reduced $(8.8 \pm 2.8,8.4 \pm 2.1$, and $9.0 \pm 3.4 \mathrm{mmol} / \mathrm{L}$ in Group 1, Group 2 and control group, respectively). However, by the end of the experiment it reached physiological range both in treatment and control groups. Its difference vs. the control was $4.7 \%$ in Diatomite treated group $(p<0.05)$ and $41.6 \%$ in Biokoretron treated group ( $p<0.01$ ). Thus, the use of diatomaceous earth based drugs optimizes blood iron levels.

Diatomaceous earth based drugs had a significant effect on the non-specific resistance.

The data obtained allowed revealing low level of serum bactericidal activity that was of $23.7 \pm 1.15 \%, 24.0 \pm 2.03 \%$, and $23.1 \pm 7.52 \%$ in the control group, Group 1 and Group 2, respectively.

However, as early as on Day 10 serum bactericidal activity significantly increased in treatment groups while decreasing in the control group. On Day 20, serum bactericidal activity level increased 1.7-, 2.2- and 2.4-fold in the control group, Group 1 (Diatomite) and Group 2 (Biokoretron) vs. baseline, respectively. These findings were statistically significant ( $p \leq 0.01$ or $p \leq 0.001$ ). This is due to the fact that all the treated and control pigs were administered with an antibiotic drug in order to prevent intestinal infections. It is known that antibiotics affect the level of serum bactericidal activity that resulted in increased parameters.

By the end of the experiment, all groups of animals demonstrated a decrease in serum bactericidal activity levels. On Day 30, pigs were 2--2.5 month old, and might be already affected by aging changes resulting in decreased activity of natural resistance that is balanced out by specific immunity stimulation. The non-specific resistance is known to be most active in the first months of life, while the specific immunity is formed 
and reinforced later on because of contact with environment as well as due to the planned vaccinations.

The data obtained shows that the pigs in treatment groups had 1.6-fold (Biokoretron) and 1.3-fold (Diatomite) higher bactericidal activity vs. the control counterparts. Therefore, study of serum bactericidal activity revealed a clear trend of superiority of Groups 1 and 2 over the control group.

The serum lysozyme activity at the baseline was of $34.1 \pm 3.0 \%$ in Group 1, $33.2 \pm$ $3.4 \%$ in Group 2, and $34.8 \pm 3.3 \%$ in control group.

By Day 10, serum lysozyme activity in control group decreased by $5.6 \%$. However, it increased 2.1- and 5.2-fold in Groups 1 and 2, respectively. The decrease in serum lysozyme activity in the control group was associated with stress due to in-process movements within the pig farm. On Day 20, all the groups demonstrated a sharp decline in the blood lysozyme levels. The decline was the least in the group receiving Biokoretron, lysozyme activity in this group was 1.9-fold higher than in the control group ( $p \leq 0.01$ ). Diatomite treated group had 1.4-fold difference with a control group. The decrease in serum lysozyme activity in the treatment groups was associated with stress due to in-process movements within the pig farm. It is known that the effect of stress factors is the most common in an exposition to a number of etiological factors suppressing both total immunity and its individual components and may cause serious complications depending on the effect degree. The use of test drugs in treatment groups allowed not only maintaining but also increasing in the lysozyme level vs. baseline.

At the end of the experiment, the highest level of lysozyme activity was in the group comprising Biokoretron treated pigs (39.5 $\pm 1.2 \%)$. In Diatomite treated group, the lysozyme activity was of $34.2 \pm 3.5 \%$ vs. $31.2 \pm 1.1 \%$ in the control group.

Thus, by the end of the experiment the lysozyme activity level in Group 2 (Biokoretron) increased 1.2-fold compared to the baseline values. No changes were observed in Group 1 (Diatomite) vs. baseline. In the control group, serum lysozyme activity declined. Thus, the use of Biokoretron and Diatomite stabilized serum lysozyme activity, while neutralizing the severity of external pathological factors.

Therefore, Biokoretron and Diatomite upregulate non-specific immunity factors. The test drugs stabilize and stimulate serum bactericidal and lysozyme activities in technological and alimentary stresses, as well as when using antibiotics. Thus, they mitigate the effect of negative factors on the pig body.

The complex effect of drugs on the various functional components of body is associated with their mechanism of action. Diatomite based drugs primarily affect the gastrointestinal tract along its entire length. When moving on the gastrointestinal tract, 
the adsorbent enhances intestinal motility and intestinal contents evacuation due to receptor irritation. Specific structure of the adsorbent crystal lattice enables sorption of toxins in the bowel lumen. The mineral contains a number of macronutrients and trace elements, which may be exchanged with intestinal content, thereby improving the intestine functions and directly affecting metabolism after intake. All above effects increase digestibility and absorption of feed nutrients, improve metabolism rate and metabolic activity of internal organs. A probiotic included in Biokoretron has a beneficial effect on the intestinal microflora, reduces the number of opportunistic strains and increases the amount of saprophytic microflora.

\section{Conclusion}

Diatomaceous earth-based drugs, Biokoretron and Diatomite, have growth promoting and antianemic effects on the weaned pigs, increase the protein and mineral metabolism rate, and stimulate non-specific immunity factors.

\section{Conflict of Interest and Ethical Approval}

The authors declare that they have no conflict of interest.

This article does not contain any studies with human participants performed by any of the authors. All applicable international, national, and/or institutional guidelines for the care and use of animals were followed.

\section{References}

[1] Semenenko, M.P., Antipov, V.A., Savinkov, A.V. et al. (2010). Veterinaria Kubani, vol. 6, pp. 33--34

[2] Semenenko, M.P., Antipov, V.A., Matiushevskij, L.A., Fontanetckij, A.S., Tjapkina, E.V. (2009). Krasnodar: Krasnodar RVI, 249 p.

[3] Pokhodnja, G.S., Shaposhnikov, A.A., Manokhina, L.A. et al. (2005). In: Problems of Animal Husbandry, vol. 5, pp. 95--97. Belgorod: Belgorod SAA.

[4] Attia, Y.A., Allakany, H.F., Abd Al-Hamid, A.E. et al. (2012). Journal of animal physiology and animal nutrition vol. 97, pp. 754--772.

[5] Chittavong, M., Lindberg, J.E., Jansson A. (2013). Trop. Anim. Health Prod., no. 45, pp. 505--510. 
[6] Malkov, A.A., Belko, A.A., Velikanov, V.V. et al. (2010). Proceedings of Vitebsk State Academy of Veterinary Medicine, vol. 46, pp. 41--44.

[7] Sevastjanova, N.A. (2001). Scientific and Production Conference on Current Issues in Veterinary Medicine and Animal Sciences. Kazan, pp. 178--179.

[8] Vasilevskaja, E.M., Velikanov, V.V., Aleshkevich, V.N. (2014). Proceedings of Vitebsk State Academy of Veterinary Medicine, vol. 50, pp. 93--95.

[9] Denli, M., Blandon, J.C., Guynot, M.E. Salado, S., Perez J.F. (2015). Journal Anim. Sci., vol. 93, pp. 637--645.

[10] Dos Anjos, F.R., Ledoux, D.R., Rottinghaus, G.E., Chimonyo, M. (2015). Br. Poult. Sci., vol. 56, pp. 459--469.

[11] Eckhardt, J.C. et al. (2014). Br. Poult. Sci., vol. 55, pp. 215--220. 